University of Wollongong

Research Online

Faculty of Engineering - Papers (Archive)

Faculty of Engineering and Information

Sciences

24-1-2005

\title{
Nonlinear response formula for an interacting two-dimensional electron gas under a magnetic field and microwave radiation
}

T. Toyoda

Tokai University, Japan

M. Fujita

Tokai University, Japan

H. Koizumi

Nippon Gear Co., Kanagawa, Japan

C. Zhang

University of Wollongong, czhang@uow.edu.au

Follow this and additional works at: https://ro.uow.edu.au/engpapers

Part of the Engineering Commons

https://ro.uow.edu.au/engpapers/226

\section{Recommended Citation}

Toyoda, T.; Fujita, M.; Koizumi, H.; and Zhang, C.: Nonlinear response formula for an interacting twodimensional electron gas under a magnetic field and microwave radiation 2005.

https://ro.uow.edu.au/engpapers/226

Research Online is the open access institutional repository for the University of Wollongong. For further information contact the UOW Library: research-pubs@uow.edu.au 


\title{
Nonlinear response formula for an interacting two-dimensional electron gas under a magnetic field and microwave radiation
}

\author{
Tadashi Toyoda, ${ }^{1}$ Maho Fujita, ${ }^{1}$ Hideki Koizumi, ${ }^{2}$ and Chao Zhang $^{3}$ \\ ${ }^{1}$ Department of Physics, Tokai University, Kitakaname 1117, Hiratsuka, Kanagawa, Japan 259-1292 \\ ${ }^{2}$ Nippon Gear Co., Ltd., Kirihara-cho 7, Fujisawa, Kanagawa, Japan 252-0811 \\ ${ }^{3}$ School of Engineering Physics, University of Wollongong, New South Wales 2522, Australia
}

(Received 22 October 2004; published 24 January 2005)

\begin{abstract}
Introducing a canonical transformation of the second quantized field operators for two-dimensional interacting electrons under a uniform static magnetic field and a microwave radiation field, an exact formula that transforms the retarded density response function of the electrons to that of the electrons without the radiation field is derived. The formula provides a new way to treat the effects of the microwave radiation field on the electron density response when the conventional linear response approximation is not applicable.
\end{abstract}

DOI: 10.1103/PhysRevB.71.033313

PACS number(s): 73.20.Mf, 73.50.Bk, 73.50.Fq, 05.30.Fk

In recent experiments, it was observed that high mobility electrons moving under a uniform static magnetic field and a microwave radiation can exhibit additional magnetic oscillations [1-3]. Furthermore, due to the interplay between the wavelength of the microwave radiation $(\lambda)$ and the electron magnetic length $(l)$, the dc resistivity vanishes at some particular ratio of $l / \lambda$. This observation demonstrates that resonant scattering of the two-dimensional electrons under the radiation field can realize transport with zero energy loss. The discovery of the zero resistance state has led to renewed theoretical interests in the electronic properties under radiation. The well-established linear response approximation in quantum many-body theory seems to fail in the resonant coupling regime and a new theoretical method is sought after. In this paper we shall present a quantum field theoretical canonical transformation to treat the nonlinear response of an interacting two-dimensional electrons subjected to an intense radiation field and a uniform static magnetic field simultaneously.

We assume the two-dimensional electrons are described by the second quantized Schrödinger field operators $\hat{\Psi}_{\alpha}(\mathbf{r}, t)$ and $\hat{\Psi}_{\beta}^{\dagger}(\mathbf{r}, t)$ that satisfy the equal-time canonical anticommutation relation,

$$
\hat{\Psi}_{\alpha}(\mathbf{r}, t) \hat{\Psi}_{\beta}^{\dagger}\left(\mathbf{r}^{\prime}, t\right)+\hat{\Psi}_{\beta}^{\dagger}\left(\mathbf{r}^{\prime}, t\right) \hat{\Psi}_{\alpha}(\mathbf{r}, t)=\delta_{\alpha \beta} \delta\left(\mathbf{r}-\mathbf{r}^{\prime}\right),
$$

and the Hamiltonian is composed of three parts, i.e., the kinetic energy term $H_{A}$, the spin coupling term $H_{S}$ and the electron-electron many-body interaction term $H_{\text {int }}$. The kinetic energy term is

$$
H_{A}=\int d^{2} \mathbf{r} \sum_{\alpha} \hat{\Psi}_{\alpha}^{\dagger}(\mathbf{r}, t) \varepsilon_{A}(\boldsymbol{\nabla}) \hat{\Psi}_{\alpha}(\mathbf{r}, t),
$$

where the differential operator $\varepsilon_{A}$ is defined as

$$
\begin{aligned}
\varepsilon_{A}= & (2 m)^{-1}\left\{\left(-i \hbar \partial_{x}\right)^{2}\right. \\
& \left.+\left(-i \hbar \partial_{y}-e c^{-1} B x-e E \omega^{-1} \cos (\omega t)\right)^{2}\right\}-\mu,
\end{aligned}
$$

and $m$ is the electron mass, $-e$ is the electron charge, $\mu$ is the chemical potential and $d^{2} \mathbf{r} \equiv d x d y$. The magnetic flux density is defined as $\vec{B}=\left\{B_{x}=0, B_{y}=0, B_{z}=B\right\}$ and the electrical field amplitude of the laser radiation is defined as $\vec{E}=\left\{E_{x}=0, E_{y}\right.$ $\left.=E \cos (\omega t), E_{z}=0\right\}$. The spin coupling term $H_{S}$ is written in terms of the electron number density operator $\rho_{\alpha}^{A}(\mathbf{r}, t)$ $\equiv \hat{\Psi}_{\alpha}^{\dagger}(\mathbf{r}, t) \hat{\Psi}_{\alpha}(\mathbf{r}, t)$ as

$$
H_{S}=\frac{g \mu_{B} B}{2} \int d^{2} \mathbf{r}\left\{\rho_{\uparrow}^{A}(\mathbf{r}, t)-\rho_{\downarrow}^{A}(\mathbf{r}, t)\right\},
$$

where $g$ is the electron $g$-factor and $\mu_{B}$ is the Bohr magneton. The electron-electron interaction Hamiltonian is assumed to be

$$
\begin{aligned}
H_{i n t}= & \frac{1}{2} \sum_{\alpha, \beta} \int d^{2} \mathbf{r} \hat{\Psi}_{\alpha}^{\dagger}(\mathbf{r}, t) \hat{\Psi}_{\beta}^{\dagger}\left(\mathbf{r}^{\prime}, t\right) U_{i n t}\left(\mathbf{r}-\mathbf{r}^{\prime}\right) \\
& \times \hat{\Psi}_{\beta}\left(\mathbf{r}^{\prime}, t\right) \hat{\Psi}_{\alpha}(\mathbf{r}, t),
\end{aligned}
$$

where $U_{\text {int }}$ is an interaction potential. The dynamics of the field operator $\hat{\Psi}_{\alpha}$ is given by the Heisenberg equation,

$$
i \hbar \partial_{t} \hat{\Psi}_{\alpha}(\mathbf{r}, t)=\left[\hat{\Psi}_{\alpha}(\mathbf{r}, t), H_{A}(t)+H_{S}(t)+H_{\text {int }}(t)\right] .
$$

By making use of the explicit form of the Hamiltonian and the equal-time canonical anti-commutation relation we can readily obtain

$$
\begin{aligned}
i \hbar \partial_{t} \hat{\Psi}_{\alpha}(\mathbf{r}, t)= & \varepsilon_{A}(\boldsymbol{\nabla}) \hat{\Psi}_{\alpha}(\mathbf{r}, t)+\sigma(\alpha) \frac{g \mu_{B}}{2} B \hat{\Psi}_{\alpha}(\mathbf{r}, t) \\
& +\sum_{\beta} \int d^{2} \mathbf{r}^{\prime} \hat{\Psi}_{\beta}^{\dagger}\left(\mathbf{r}^{\prime}, t\right) \hat{\Psi}_{\beta}\left(\mathbf{r}^{\prime}, t\right) \\
& \times U_{\text {int }}\left(\mathbf{r}^{\prime}-\mathbf{r}\right) \hat{\Psi}_{\alpha}(\mathbf{r}, t),
\end{aligned}
$$

where we have defined $\sigma(\uparrow)=1$ and $\sigma(\downarrow)=-1$. It should be noted here that this is an equation for the second quantized field operator. The quantum many-body effects come into the dynamics through the nonlinear term on the right-hand side of (7). In Ref. 4 the Schrödinger equation for a singleelectron wave function was considered and a quantum mechanical canonical transformation to eliminate the electric 
field was obtained. A similar transformation may be constructed for the second quantized field operator for the interacting many-electron system,

$$
\hat{\Psi}_{\alpha}(\mathbf{r}, t)=e^{(i / \hbar) \Lambda(\mathbf{r}, t)} \hat{\Phi}_{\alpha}(\mathbf{r}+\mathbf{u}(t), t) .
$$

This defines a new second quantized field operator $\hat{\Phi}_{\alpha}$ provided that the phase function $\Lambda$ and the displacement $\mathbf{u}$ are specified. Because these are $c$-number functions, it is straightforward to see that the equal-time canonical anticommutation relation also holds for the transformed field operators: 5

$$
\hat{\Phi}_{\alpha}(\mathbf{r}, t) \hat{\Phi}_{\beta}^{\dagger}\left(\mathbf{r}^{\prime}, t\right)+\hat{\Phi}_{\beta}^{\dagger}\left(\mathbf{r}^{\prime}, t\right) \hat{\Phi}_{\alpha}(\mathbf{r}, t)=\delta_{\alpha \beta} \delta\left(\mathbf{r}-\mathbf{r}^{\prime}\right) .
$$

Therefore, the transformation from $\hat{\Psi}_{\alpha}$ to $\hat{\Phi}_{\alpha}$ is canonical. Since the functional form of the linear part of the field equation for $\hat{\Psi}_{\alpha}$ is equivalent to that of the Schrödinger equation for the single-electron wave function, it would be quite natural to expect a transformation similar to the unitary transformation for the wave function introduced in Ref. 4 might work as well. If we assume $\Lambda=u_{1}(t)+u_{2}(t) x, u_{x}=u_{3}(t)$, and $u_{y}(t)=u_{5}(t)$ and substitute the transformation (8) into the field equation (7), the left-hand side of the field equation yields

$$
\begin{aligned}
i \hbar \partial_{t} \hat{\Psi}_{\alpha}(\mathbf{r}, t)= & e^{(i / \hbar)\left(u_{1}+u_{2} x^{\prime}-u_{2} u_{3}\right)}\left[-\dot{u}_{1}-\dot{u}_{2} x^{\prime}+\dot{u}_{2} u_{3}+i \hbar \dot{u}_{3} \partial_{x^{\prime}}\right. \\
& \left.+i \hbar \dot{u}_{5} \partial_{y^{\prime}}+i \hbar \partial_{t}\right] \hat{\Phi}_{\alpha}\left(x^{\prime}, y^{\prime}, t\right),
\end{aligned}
$$

where we have defined the variables $x^{\prime} \equiv x+u_{3}(t)$ and $y^{\prime}$ $\equiv y+u_{5}(t)$. The first term on the right-hand side of the field equation (7) can be written as

$$
\begin{aligned}
\varepsilon_{A}(\boldsymbol{\nabla}, x) \hat{\Psi}_{\alpha}(x, y, t)= & e^{(i / \hbar)\left(u_{1}+u_{2} x^{\prime}-u_{2} u_{3}\right)} \\
& \times\left[\varepsilon_{B}\left(\boldsymbol{\nabla}^{\prime}, x^{\prime}\right)+\frac{u_{2}^{2}}{2 m}+\frac{e^{2} A^{* 2}}{2 m c^{2}}+\frac{e^{2} B A^{*}}{m c^{2}} x^{\prime}\right. \\
& \left.-i \hbar \frac{u_{2}}{m} \partial_{x^{\prime}}+i \hbar \frac{e A^{*}}{m c} \partial_{y^{\prime}}\right] \hat{\Phi}_{\alpha}\left(x^{\prime}, y^{\prime}, t\right),
\end{aligned}
$$

where we have defined

$$
A *(t)=E c \omega^{-1} \cos (\omega t)-B u_{3}(t)
$$

and

$$
\varepsilon_{B}\left(\nabla^{\prime}, x^{\prime}\right)=(2 m)^{-1}\left[\left(-i \hbar \partial_{x^{\prime}}\right)^{2}+\left(-i \hbar \partial_{y^{\prime}}-e c^{-1} B x^{\prime}\right)^{2}\right] .
$$

The spin term on the right-hand side of (7) is transformed to $\sigma(\alpha) \frac{g \mu_{B}}{2} B \hat{\Psi}_{\alpha}(\mathbf{r}, t)=e^{(i / \hbar)\left(u_{1}+u_{2} x^{\prime}-u_{2} u_{3}\right)} \sigma(\alpha) \frac{g \mu_{B}}{2} B \hat{\Phi}_{\alpha}\left(x^{\prime}, y^{\prime}, t\right)$.

After substituting the transformation (8), the electronelectron interaction term on the right-hand side of the field equation (7) can be written as

$$
\begin{aligned}
\sum_{\beta} \int & d^{2} \mathbf{r}^{\prime \prime} \hat{\Psi}_{\beta}^{\dagger}\left(\mathbf{r}^{\prime \prime}, t\right) \hat{\Psi}_{\beta}\left(\mathbf{r}^{\prime \prime}, t\right) U_{i n t}\left(\mathbf{r}^{\prime \prime}-\mathbf{r}\right) \hat{\Psi}_{\alpha}(\mathbf{r}, t) \\
= & e^{(i / \hbar)\left(u_{1}+u_{2} x^{\prime}-u_{2} u_{3}\right)} \sum_{\beta} \int d^{2} \mathbf{r}^{\prime \prime} \hat{\Phi}_{\beta}^{\dagger}\left(\mathbf{r}^{\prime \prime}, t\right) \hat{\Phi}_{\beta}\left(\mathbf{r}^{\prime \prime}, t\right) \\
& \times U_{i n t}\left(\mathbf{r}^{\prime \prime}-\mathbf{r}^{\prime}\right) \hat{\Phi}_{\alpha}\left(\mathbf{r}^{\prime}, t\right),
\end{aligned}
$$

where we have made the change of variables in the integration and also defined $\mathbf{r}^{\prime} \equiv\left(x^{\prime}, y^{\prime}\right)$. Now we put together (10), (11), (14), and (15), and require that the variables $u_{1}, u_{2}, u_{3}$ and $u_{5}$ satisfy the equations

$$
\begin{gathered}
\dot{u}_{2} u_{3}-\dot{u}_{1}=(2 m)^{-1} u_{1}^{2}+\left(2 m c^{2}\right)^{-1} e^{2} A^{* 2}, \\
-\dot{u}_{2}=e^{2}\left(m c^{2}\right)^{-1} B A * \\
-\dot{u}_{5}=-e(m c)^{-1} A *,
\end{gathered}
$$

and

$$
-\dot{u}_{3}=m^{-1} u_{2}
$$

Then the field equation for $\hat{\Phi}_{\alpha}\left(x^{\prime}, y^{\prime}, t\right)$ reduces to

$$
\begin{aligned}
i \hbar \partial_{t} \hat{\Phi}_{\alpha}\left(\mathbf{r}^{\prime}, t\right)= & \varepsilon_{B}\left(\nabla^{\prime}, x^{\prime}\right) \hat{\Phi}_{\alpha}\left(\mathbf{r}^{\prime}, t\right)+\sigma(\alpha) \frac{g \mu_{B}}{2} B \hat{\Phi}_{\alpha}(\mathbf{r}, t) \\
& +\sum_{\beta} \int d^{2} \mathbf{s}^{\prime} \hat{\Phi}_{\beta}^{\dagger}\left(\mathbf{s}^{\prime}, t\right) \hat{\Phi}_{\beta}\left(\mathbf{s}^{\prime}, t\right) \\
& \times U_{\text {int }}\left(\mathbf{s}^{\prime}-\mathbf{r}^{\prime}\right) \hat{\Phi}_{\alpha}\left(\mathbf{r}^{\prime}, t\right) .
\end{aligned}
$$

Obviously this equation shows that the field operator $\hat{\Phi}_{\alpha}$ describes the electrons moving in the magnetic field only and the time-dependent electromagnetic field has been eliminated. It should be noted here that the transformation (8) does not affect the electron-electron interaction term in the field equation for $\hat{\Phi}_{\alpha}$. This is the beauty of the field theoretical canonical transformation. ${ }^{5}$ In the quantum mechanical unitary transformation for the single-electron wave function, it is not possible to include the many-electron interaction. The equations (16)-(19) can be easily solved. ${ }^{4}$ For the initial condition

$$
u_{1}(0)=u_{2}(0)=u_{3}(0)=u_{5}(0)=0,
$$

the solutions are

$$
\begin{aligned}
& u_{1}(t)= \frac{e^{2} E^{2}}{2 m}\left(\frac{1}{\omega_{c}^{2}-\omega^{2}}\right)^{2}\left[\frac{\omega_{c}^{3}}{2 \omega^{2}} \sin \left(2 \omega_{c} t\right)+\frac{\omega}{4}\left(\frac{3 \omega_{c}^{2}}{\omega^{2}}-1\right)\right. \\
& \times \sin (2 \omega t)+\left(\frac{\omega_{c}^{2}-\omega^{2}}{2}\right) t-\frac{\omega_{c}^{3}}{\omega^{2}}\left\{\sin \left[\left(\omega_{c}-\omega\right) t\right]\right. \\
&\left.\left.+\sin \left[\left(\omega_{c}+\omega\right) t\right]\right\}\right] \\
& u_{2}(t)=e E\left(\frac{\omega_{c}}{\omega_{c}^{2}-\omega^{2}}\right)\left[\frac{-\omega_{c}}{\omega} \sin \left(\omega_{c} t\right)+\sin (\omega t)\right]
\end{aligned}
$$




$$
\begin{aligned}
& u_{3}(t)=\frac{e E}{m} \frac{\omega_{c}}{\omega}\left(\frac{1}{\omega_{c}^{2}-\omega^{2}}\right)\left[-\cos \left(\omega_{c} t\right)+\cos (\omega t)\right], \\
& u_{5}(t)=\frac{e E}{m}\left(\frac{1}{\omega_{c}^{2}-\omega^{2}}\right)\left[\frac{\omega_{c}}{\omega} \sin \left(\omega_{c} t\right)-\sin (\omega t)\right],
\end{aligned}
$$

where $\omega_{c}$ is the cyclotron frequency $\omega_{c}=e B / m c$.

One of the interesting applications is to examine the effects of the oscillating electric field on the electron charge fluctuation. In the quantum mechanical formulation, ${ }^{4}$ the time-dependent perturbation was used to calculate the change of the electron wave function. As we are dealing with the second quantized field operators in the present formulation, the discussion of the charge fluctuation becomes much simpler. The operator part of the retarded electron density response function is defined such that

$$
\hat{D}_{\alpha \beta}^{A}\left(\mathbf{r}, \mathbf{r}^{\prime \prime} ; t, t^{\prime \prime}\right)=-i \theta\left(t-t^{\prime \prime}\right)\left[\rho_{\alpha}^{A}(\mathbf{r}, t), \rho_{\beta}^{A}\left(\mathbf{r}^{\prime \prime}, t^{\prime \prime}\right)\right] .
$$

Under the transformation (8) the electron number density $\rho_{\alpha}^{A}(\mathbf{r}, t)$ is expressed as

$$
\rho_{\alpha}^{A}(\mathbf{r}, t)=\hat{\Phi}_{\alpha}^{\dagger}(\mathbf{r}+\mathbf{u}(t), t) \hat{\Phi}_{\alpha}(\mathbf{r}+\mathbf{u}(t), t) \equiv \rho_{\alpha}^{B}(\mathbf{r}+\mathbf{u}(t), t) .
$$

If we define the operator part of the density-density retarded response function for the electrons without the electric field,

$$
\hat{D}_{\alpha \beta}^{B}\left(\mathbf{r}, \mathbf{r}^{\prime \prime} ; t, t^{\prime \prime}\right)=-i \theta\left(t-t^{\prime \prime}\right)\left[\rho_{\alpha}^{B}(\mathbf{r}, t), \rho_{\beta}^{B}\left(\mathbf{r}^{\prime \prime}, t^{\prime \prime}\right)\right],
$$

then the two operators, $\hat{D}_{\alpha \beta}^{A}$ and $\hat{D}_{\alpha \beta}^{B}$, are related as

$$
\hat{D}_{\alpha \beta}^{A}\left(\mathbf{r}, \mathbf{r}^{\prime \prime} ; t, t^{\prime \prime}\right)=\hat{D}_{\alpha \beta}^{B}\left(\mathbf{r}+\mathbf{u}(t), \mathbf{r}^{\prime \prime}+\mathbf{u}\left(t^{\prime \prime}\right) ; t, t^{\prime \prime}\right) .
$$

If we define the initial ground state expectation value $\langle\cdots\rangle$ for the operators, then the response functions are written as

$$
\begin{aligned}
D_{\alpha \beta}^{A}\left(\mathbf{r}, \mathbf{r}^{\prime \prime} ; t, t^{\prime \prime}\right) & =\left\langle\hat{D}_{\alpha \beta}^{A}\left(\mathbf{r}, \mathbf{r}^{\prime \prime} ; t, t^{\prime \prime}\right)\right\rangle \\
& =-i \theta\left(t-t^{\prime \prime}\right)\left\langle\left[\rho_{\alpha}^{A}(\mathbf{r}, t), \rho_{\beta}^{A}\left(\mathbf{r}^{\prime \prime}, t^{\prime \prime}\right)\right]\right\rangle
\end{aligned}
$$

and

$$
\begin{aligned}
D_{\alpha \beta}^{B}\left(\mathbf{r}, \mathbf{r}^{\prime \prime} ; t, t^{\prime \prime}\right) & =\left\langle\hat{D}_{\alpha \beta}^{B}\left(\mathbf{r}, \mathbf{r}^{\prime \prime} ; t, t^{\prime \prime}\right)\right\rangle \\
& =-i \theta\left(t-t^{\prime \prime}\right)\left\langle\left[\rho_{\alpha}^{B}(\mathbf{r}, t), \rho_{\beta}^{B}\left(\mathbf{r}^{\prime \prime}, t^{\prime \prime}\right)\right]\right\rangle .
\end{aligned}
$$

Since these response functions have the translational invariance, ${ }^{6,7}$ i.e., $D_{\alpha \beta}^{A}=D_{\alpha \beta}^{A}\left(\mathbf{r}-\mathbf{r}^{\prime \prime} ; t, t^{\prime \prime}\right)$ and $D_{\alpha \beta}^{B}=D_{\alpha \beta}^{B}(\mathbf{r}$ $\left.-\mathbf{r}^{\prime \prime} ; t, t^{\prime \prime}\right)$, we obtain the formula

$$
\begin{aligned}
D_{\alpha \beta}^{A}\left(x-x^{\prime \prime}, y-y^{\prime \prime} ; t, t^{\prime \prime}\right)= & D_{\alpha \beta}^{B}\left(x-x^{\prime \prime}+u_{3}(t)\right. \\
& \left.-u_{3}\left(t^{\prime \prime}\right), y-y^{\prime \prime}+u_{5}(t)-u_{5}\left(t^{\prime \prime}\right) ; t, t^{\prime \prime}\right) .
\end{aligned}
$$

This is the principal new result of this work. Once the density response function $D_{\alpha \beta}^{B}$ is calculated, the effects of the oscillating electric field on the response function can be examined by this transformation. It should be remarked that this formula can be used to investigate nonlinear effects with respect to the time-dependent electric field, i.e., the effects of an intense microwave radiation field acting on the electrons.
Although the above formulas are for zero temperature, the finite temperature effects can be included by replacing the ground state expectation value $\langle\cdots\rangle$ by an appropriate grand canonical ensemble expectation value.

As an example of the application of the theory, we calculate the electronic polarizability and the dieclectric response function. The electronic polarizabilty $\Pi_{B}\left(\mathbf{r}, \mathbf{r}^{\prime} ; t, t^{\prime}\right)$ is given as

$$
\begin{aligned}
\Pi_{B}\left(\mathbf{r}, \mathbf{r}^{\prime} ; t, t^{\prime}\right)= & -2 i \sum_{n k_{y}} \sum_{n^{\prime} k_{y}^{\prime}} u_{n^{\prime} k_{y}^{\prime}}^{*}(\mathbf{r}, t) u_{n k_{y}}(\mathbf{r}, t) \\
& \times u_{n k_{y}}^{*}\left(\mathbf{r}^{\prime}, t^{\prime}\right) u_{n^{\prime} k_{y}^{\prime}}\left(\mathbf{r}^{\prime}, t^{\prime}\right)\left[f_{n, k_{y}}-f_{n^{\prime}, k_{y}^{\prime}}\right],
\end{aligned}
$$

where $u_{n k_{y}}$ 's are Landau orbitals and $f$ is the Fermi-Dirac distribution function. Now we shift the coordinate due to the coupling to the radiation field, then perform Fourier transform. Integrating over $y$ results $k_{y}^{\prime}=k_{y}+q_{y}$ and $q_{y}=q_{y}^{\prime}$. The polarizability can be further written as

$$
\begin{aligned}
\Pi_{A}\left(\mathbf{q}, \mathbf{q}^{\prime} ; t, t^{\prime}\right)= & -2 i \sum_{n k_{y}} \sum_{n^{\prime}} M_{n n^{\prime}}\left(q_{x}, k_{y}, k_{y}+q_{y} ; t\right) M_{n n^{\prime}} \\
& \times\left(-q_{x}^{\prime}, k_{y}, k_{y}-q_{y} ; t^{\prime}\right) \exp \left\{i \frac{\gamma_{0} q_{y}}{\omega_{c}^{2}-\omega^{2}}[\cos \omega t\right. \\
& \left.\left.-\cos \omega t^{\prime}-\cos \omega_{c} t-\cos \omega_{c} t^{\prime}\right]\right\} \\
& \times\left[f_{n, k_{y}}-f_{n^{\prime}, k_{y}+q_{y}}\right] e^{-i\left(E_{n}-E_{n^{\prime}}\right)\left(t-t^{\prime}\right) / \hbar} .
\end{aligned}
$$

Here

$$
\begin{aligned}
M_{n n^{\prime}}\left(q_{x}, k_{y}, k_{y}+q_{y}\right)= & \sqrt{\frac{n_{<}}{n_{>}}} i^{n_{>}-n_{<}} \exp \left[i q_{x} l^{2}\left(k_{y}+q_{y} / 2\right)\right. \\
& \left.-i q_{x} 2 u_{3}(t)\right] \exp (-Y / 2) \\
& \times Y^{\left(n_{>}-n_{<}\right) / 2} L_{n_{<}}^{n_{>}-n_{<}}(Y) \\
= & \exp \left[i q_{x} l^{2} k_{y}\right] N_{n n^{\prime}}\left(q_{x}, q_{y}\right)
\end{aligned}
$$

where $Y=(1 / 2)(q l)^{2}$. Substituting (35) in (34) and summing over $k_{y}$ gives $q_{x}^{\prime}=q_{x}$. The result that $\mathbf{q}=\mathbf{q}^{\prime}$ shows that system's translational invariance is not affected by a perpendicular magnetic field and a long wavelength electromagnetic radiation. Now we first expand the exponential function according to the generating function of the Bessel function, $\exp (i \alpha \cos \omega t)=\Sigma_{m} i^{m} J_{m}(\alpha) \exp (i m \omega t)$, then we introduce $\tau$ $=t-t^{\prime}$ and $T=\left(t+t^{\prime}\right) / 2$. After averaging over $T$ for one period of the radiation field and performing the Fourier transform in $\tau$, we obtain

$$
\begin{aligned}
\Pi_{A}(\mathbf{q} ; \Omega)= & -2 i \sum_{n n^{\prime}} N_{n n^{\prime}}\left(q_{x}, q_{y}\right) N_{n n^{\prime}}\left(q_{x}, q_{y}\right) \sum_{m m^{\prime}} J_{m}^{2}\left(\gamma_{0} q_{y}\right) \\
& \times J_{m^{\prime}}^{2}\left(\gamma_{0} q_{y}\right) \frac{f_{n, k_{y}}-f_{n^{\prime}, k_{y}+q_{y}}}{\Omega-\left(E_{n}-E_{n^{\prime}}\right) / \hbar-m \omega-m^{\prime} \omega_{c}} .
\end{aligned}
$$

The dielectric function can now also calculated. Within 
the random phase approximation, the inverse dielectric function is given by

$$
\begin{aligned}
\frac{1}{\epsilon\left(\mathbf{q} ; t, t^{\prime}\right)}= & \delta\left(t-t^{\prime}\right)+V_{q} \Pi\left(\mathbf{q} ; t, t^{\prime}\right) \\
& +V_{q}^{2} \int d t_{1} \Pi\left(\mathbf{q} ; t, t_{1}\right) \Pi\left(\mathbf{q} ; t_{1}, t^{\prime}\right)+\cdots .
\end{aligned}
$$

Here again the translational invariance is used to obtain the dielectric response in $\mathbf{q}$-space, $V_{q}=2 \pi e^{2} / q$ is the Coulomb interaction. We again average over $T$ and perform the Fourier transform for $\tau$. The inverse dielectric function is given as

$$
\frac{1}{\epsilon(q, \Omega)}=\sum_{m, m^{\prime}} \frac{J_{m}^{2}\left(q_{y} \gamma_{0}\right) J_{m^{\prime}}^{2}\left(q_{y} \gamma_{0}\right)}{1-V_{q} Q\left(q, \Omega-m \omega-m^{\prime} \omega_{c}\right)},
$$

where $Q(q, \Omega)$ is the electronic polarizability of the system in the absence of the $\mathrm{THz}$ laser field,

$$
Q(q, \tau)=-2 i \sum_{n n^{\prime}} N_{n n^{\prime}}\left(q_{x}, q_{y}\right) N_{n n^{\prime}}\left(q_{x}, q_{y}\right)\left[f_{n}-f_{n^{\prime}}\right] e^{-i\left(E_{n}-E_{n^{\prime}}\right) \tau / \hbar} .
$$

The above result is directly related to the light scattering cross-section, $d \sigma / d \Omega \propto \Im m[1 / \epsilon(q, \Omega)]$. In the absence of finite Landau level broadening, only collective excitations contribute to the scattering cross-section,

$$
\begin{aligned}
\Im m\left[\frac{1}{\epsilon(q, \Omega)}\right]= & \sum_{m, m^{\prime}} J_{m}^{2}\left(q_{y} \gamma_{0}\right) J_{m^{\prime}}^{2}\left(q_{y} \gamma_{0}\right) \delta\left[1-V_{q} Q(q, \Omega+m \omega\right. \\
& \left.\left.+m^{\prime} \omega_{c}\right)\right] .
\end{aligned}
$$

It can be seen that the spectral weight located in the principal plasmon mode $\left(\Omega_{p}\right)$ in the absence of the radiation field is now distributed in a series of photon induced plasmon modes, $\Omega\left(m, m^{\prime}\right)=\omega_{p}+m \omega+m^{\prime} \omega_{c}$. The oscillator strength of each mode is proportional to $J_{m}^{2}\left(q_{y} \gamma_{0}\right) J_{m^{\prime}}^{2}\left(q_{y} \gamma_{0}\right)$. This behavior is shown in Fig. 1 where the $x$-axis is the reduced field intensity, $r=k_{F} e E /\left(m *(\mathrm{THz})^{2}\right)$. We used parameters for GaAs-based semiconductors, $m *=0.067 m_{0}, r_{s}=m * e^{2} / \epsilon_{0} K_{f}$ $=1.0, \omega=1 \mathrm{THz}, \omega_{c}=0.9 \mathrm{THz}$. For small $q$ and lower radiation intensity, the spectral is dominantly located at $\Omega(0,0)$.

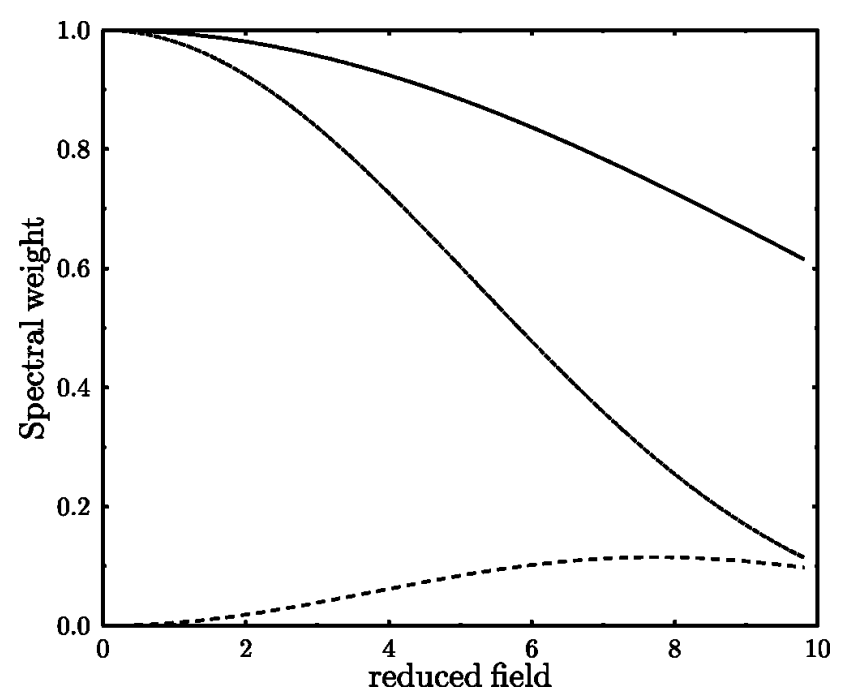

FIG. 1. A plot of spectral weight of light scattering cross section of two lowest plasmon modes. The solid line is for $\Omega(0,0)$ at $q$ $=0.1$, the dotted line is for $\Omega(0,0)$ at $q=0.2$, the broken line is for $\Omega(1,0)$ at $q=0.2$. The other parameters are given in the text.

As both $q$ and $r$ increase, a significant amount of the spectral weight shifts to the high order photon induced mode.

In conclusion, we have shown that the transformation proposed in Ref. 4 can be rigorously extended to an interacting electron gas by dealing with the second quantized Schrödinger field describing the electrons. The fact that the transformation (8) does not affect the electron-electron interaction term in the field equation seems very important in practical calculations, particularly in the calculation of the density response function. The essential advantage of this formulation is that one can still use the existing wellcontrolled approximation methods to calculate $D_{\alpha \beta}^{B}$, for example, the random-phase-approximation, $t$-matrix approximation or the local-field approximation. ${ }^{8}$ The applications of $D_{\alpha \beta}^{B}$ in the two-dimensional systems are abundant. It can be used to derive the optical properties of the system. The spectra of magneto-collective excitations for two-dimensional systems under an intense microwave radiation can also be obtained.
${ }^{1}$ R. G. Mani, J. H. Smet, K. von Klitzing, V. Narayanamurti, W. B. Johnson, and V. Umansky, Nature (London) 420, 646 (2002).

${ }^{2}$ M. A. Zudov, R. R. Du, J. A. Simmons, and J. R. Reno, Phys. Rev. B 64, 201311(R) (2001).

${ }^{3}$ M. A. Zudov, R. R. Du, L. N. Pfeiffer, and K. W. West, Phys. Rev. Lett. 90, 046807 (2003).

${ }^{4}$ M. Fujita, T. Toyoda, J. C. Cao, and C. Zhang, Phys. Rev. B 67,
075105 (2003).

${ }^{5}$ T. Toyoda and H. Koizumi, J. Phys. A 31, 6209 (1998).

${ }^{6}$ T. Toyoda, Y. Takahashi, and C. Zhang, Phys. Rev. B 50, 18640 (1994).

${ }^{7}$ K. Ito, M. Fukuda, and T. Toyoda, Phys. Rev. B 56, 10161 (1997).

${ }^{8}$ C. Zhang and G. Gumbs, Ann. Phys. (N.Y.) 224, 193 (1993). 